\title{
NEW FAMILIES OF INTEGER MATRICES WHOSE LEADING PRINCIPAL MINORS FORM SOME WELL-KNOWN SEQUENCES*
}

\author{
ALI REZA MOGHADDAMFAR ${ }^{\dagger}$, KAMBIZ MOGHADDAMFAR ${ }^{\ddagger}$, AND HADISEH \\ TAJBAKHSH ${ }^{\ddagger}$
}

\begin{abstract}
The purpose of this article is to obtain some new infinite families of Toeplitz matrices, 7-matrices and generalized Pascal triangles whose leading principal minors form the Fibonacci, Lucas, Pell and Jacobsthal sequences. We also present a new proof for Theorem 3.1 in [R. Bacher. Determinants of matrices related to the Pascal triangle. J. Théor. Nombres Bordeaux, 14:19-41,
\end{abstract} 2002.].

Key words. Fibonacci sequence, Lucas sequence, Pell sequence, Jacobsthal sequence, Determinant, Toeplitz matrix, 7-matrix, Generalized Pascal triangle, Matrix factorization, Recurrence relation.

AMS subject classifications. 15A09, 15A36, 11C20.

\section{In memory of Professor Michael Neumann.}

1. Introduction and notation. Throughout this article, unless noted otherwise, we will use the following notation (see for instance $[1,10]$ ):

- $\alpha=\left(\alpha_{i}\right)_{i \geq 0}$ and $\beta=\left(\beta_{i}\right)_{i \geq 0}$ are two arbitrary sequences with $\alpha_{0}=\beta_{0}(:=\gamma)$.

- $P_{\alpha, \beta}(n)$ is the generalized Pascal triangle associated with the sequences $\alpha$ and $\beta$ (see [1]), which we introduce as follows. In fact, $P_{\alpha, \beta}(n)=\left(P_{i, j}\right)_{0 \leq i, j \leq n}$ is a square matrix of order $n+1$ whose $(i, j)$-entry $P_{i, j}$ obeys the following rule:

$$
P_{i, 0}=\alpha_{i}, P_{0, i}=\beta_{i} \quad \text { for } 0 \leq i \leq n \text {, and } P_{i, j}=P_{i, j-1}+P_{i-1, j} \text { for } 1 \leq i, j \leq n \text {. }
$$

- $A_{\alpha, \beta}(n)=\left(A_{i, j}\right)_{0 \leq i, j \leq n}$ is the 7-matrix associated with the sequences $\alpha$ and $\beta$

${ }^{*}$ Received by the editors on November 18, 2010. Accepted for publication on May 20, 2011. Handling Editor: Michael Neumann.

${ }^{\dagger}$ Department of Mathematics, K.N. Toosi University of Technology, PO Box 16315-1618, Tehran, Iran (moghadam@kntu.ac.ir), and School of Mathematics, Institute for Research in Fundamental Sciences (IPM), PO Box 19395-5746, Tehran, Iran (moghadam@ipm.ir). This article was prepared while the author was visiting the Institute for Research in Fundamental Sciences (IPM). It would be a pleasure to thank IPM for its hospitality and facilities.

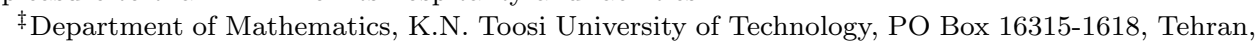
Iran. 
of order $n+1$, whose entries satisfy $A_{i, 0}=\alpha_{i}, A_{0, i}=\beta_{i}$ for $0 \leq i \leq n$, and

$$
A_{i, j}=A_{i-1, j-1}+A_{i-1, j} \text { for } 1 \leq i, j \leq n .
$$

This class of matrices was first introduced in [6].

- $L(n)=A_{(1,1,1, \ldots),(1,0,0,0, \ldots)}(n)$, which is called the unipotent lower triangular matrix of order $n+1$. If $L(n)=\left(L_{i, j}\right)_{0 \leq i, j \leq n}$, then an explicit formula for $(i, j)$-entry $L_{i, j}$ is also given by the following formula

$$
L_{i, j}=\left\{\begin{array}{cl}
0 & \text { if } \quad i<j \\
\left(\begin{array}{l}
i \\
j
\end{array}\right) & \text { if } \quad i \geq j
\end{array}\right.
$$

Moreover, we put $U(n)=L(n)^{t}$, where $A^{t}$ signifies the transpose of matrix $A$.

- $T_{\alpha, \beta}(n)=\left(T_{i, j}\right)_{0 \leq i, j \leq n}$ is the Toeplitz matrix with $T_{i, 0}=\alpha_{i}$ and $T_{0, i}=\beta_{i}$, $0 \leq i \leq n$, and $T_{i, j}=T_{k, l}$ whenever $i-j=k-l$.

- $\mathrm{R}_{i}(A)$ (resp., $\mathrm{C}_{j}(A)$ ) denotes the row $i$ (resp., column $j$ ) of matrix $A$.

- $E_{i j}$ denotes the square matrix having 1 in the $(i, j)$ position and 0 elsewhere.

- $\hat{\alpha}=\left(\hat{\alpha}_{i}\right)_{i \geq 0}$ with $\hat{\alpha}_{i}=\sum_{k=0}^{i}(-1)^{i+k}\left(\begin{array}{l}i \\ k\end{array}\right) \alpha_{k}$, which is called the binomial transform.

- $\check{\alpha}=\left(\check{\alpha}_{i}\right)_{i \geq 0}$ with $\check{\alpha}_{i}=\sum_{k=0}^{i}\left(\begin{array}{l}i \\ k\end{array}\right) \alpha_{k}$, which is called the inverse binomial transform.

- $\phi=\frac{1+\sqrt{5}}{2}$ is the golden ratio and $\Phi=\frac{1-\sqrt{5}}{2}$ is the golden ratio conjugate.

- $F_{n}$ is the $n$th Fibonacci number (A000045), which satisfies

$$
F_{0}=0, F_{1}=1, \quad F_{n}=F_{n-1}+F_{n-2} \text { for } n \geq 2 .
$$

- $L_{n}$ is the $n$th Lucas number (A000032), which satisfies

$$
L_{0}=2, L_{1}=1, \quad L_{n}=L_{n-1}+L_{n-2} \text { for } n \geq 2 \text {. }
$$

- $P_{n}$ is the $n$th Pell number (A000129), which satisfies

$$
P_{0}=0, P_{1}=1, \quad P_{n}=2 P_{n-1}+P_{n-2} \text { for } n \geq 2 .
$$

- $J_{n}$ is the $n$th Jacobsthal number (A001045), which satisfies

$$
J_{0}=0, J_{1}=1, \quad J_{n}=J_{n-1}+2 J_{n-2} \text { for } n \geq 2 .
$$

REMARK 1.1. Let $a, b, r, s$ be integers with $r, s \geq 1$. The $(a, b, r, s)$-Gibonacci sequence (or generalized Fibonacci sequence), $\left(G_{n}^{(a, b, r, s)}\right)_{n \geq 0}$, is recursively defined by: $G_{0}^{(a, b, r, s)}=a, \quad G_{1}^{(a, b, r, s)}=b \quad$ and $\quad G_{n}^{(a, b, r, s)}=r G_{n-1}^{(a, b, r, s)}+s G_{n-2}^{(a, b, r, s)}, \quad$ for $n \geq 2$. 
The Fibonacci sequence (resp., Lucas sequence, Pell sequence, or Jacobsthal sequence) correspond to the case $(a, b, r, s)=(0,1,1,1)$ (resp., $(2,1,1,1),(0,1,2,1)$ or $(0,1,1,2))$.

For convenience, some values of $F_{n}, L_{n}, P_{n}$ and $J_{n}$ for $0 \leq n \leq 13$ are determined in the following:

\begin{tabular}{l|lllllllllllllll}
$n$ & 0 & 1 & 2 & 3 & 4 & 5 & 6 & 7 & 8 & 9 & 10 & 11 & 12 & 13 & $\ldots$ \\
\hline$F_{n}$ & 0 & 1 & 1 & 2 & 3 & 5 & 8 & 13 & 21 & 34 & 55 & 89 & 144 & 233 & $\ldots$ \\
$P_{n}$ & 0 & 1 & 2 & 5 & 12 & 29 & 70 & 169 & 408 & 985 & 2378 & 5741 & 13860 & 33461 & $\ldots$ \\
$J_{n}$ & 0 & 1 & 1 & 3 & 5 & 11 & 21 & 43 & 85 & 171 & 341 & 683 & 1365 & 2731 & $\ldots$ \\
$L_{n}$ & 2 & 1 & 3 & 4 & 7 & 11 & 18 & 29 & 47 & 76 & 123 & 199 & 322 & 521 & $\ldots$
\end{tabular}

Considerable attention has been devoted in the literature to the study of symbolic evaluation of determinants (for two excellent surveys on determinant evaluations, we refer to $[8,9])$. Given an arbitrary infinite matrix $A=\left(A_{i, j}\right)_{i, j \geq 0}$, denote by $d_{n}$ the $n$th leading principal minor of $A$ defined as the determinant of the submatrix consisting of the entries in its first $n+1$ rows and columns. In this article, we are mainly interested in computation of the sequence of leading principal minors $\left(d_{0}, d_{1}, d_{2}, \ldots\right)$, especially in the case that $d_{n}$ is the $n$th Fibonacci (Lucas, Pell or Jacobsthal) number. As a matter of fact, in many papers one may encounter certain infinite matrices, the leading principal minors of which constitute a Fibonacci (sub)sequence (see for instance $[3,4,5,7,10,11])$. One of the interesting examples is the infinite matrix given by:

$$
A(\infty)=\left(A_{i, j}\right)_{i, j \geq 0}=\left(\begin{array}{rrrrrr}
1 & 1 & 1 & 1 & 1 & \cdots \\
1 & 2 & 2 & 1 & -1 & \cdots \\
1 & 4 & 6 & 6 & 3 & \cdots \\
1 & 6 & 13 & 19 & 21 & \cdots \\
1 & 10 & 25 & 45 & 66 & \cdots \\
\vdots & \vdots & \vdots & \vdots & \vdots & \ddots
\end{array}\right)
$$

Indeed, the leading principal minors of $A(\infty)$ form the Fibonacci subsequence $\left(F_{n+1}\right)_{n \geq 0}$ (see [10]). This matrix is described as follows. The first row and column are the constant sequence $(1,1,1, \ldots)$. The remaining entries $A_{i, j}$ are obtained from the following nonhomogeneous recurrence relation:

$$
A_{i, j}=A_{i, j-1}+A_{i-1, j}+i-j, \quad i, j \geq 1 .
$$

There are also other infinite integer matrices where the sequence of their leading principal minors consist of Fibonacci or Lucas numbers (see Table 1). In this table, i denotes $\sqrt{-1}$. 
Table 1. Some integer matrices with determinants as Fibonacci or Lucas numbers.

\begin{tabular}{|c|l|l|l|c|}
\hline$\gamma$ & $\alpha$ & $\beta$ & $\operatorname{Determinant}$ & Refs. \\
\hline 2 & $(2,1,1, \ldots)$ & $(2,-1,0,0, \ldots)$ & $\operatorname{det} T_{\alpha, \beta}(n)=F_{2 n+3}$ & {$[3]$} \\
2 & $(2,1,1, \ldots)$ & $(2,1,0,0, \ldots)$ & $\operatorname{det} T_{\alpha, \beta}(n)=F_{n+3}$ & {$[3]$} \\
1 & $(1, \mathbf{i}, 0,0, \ldots)$ & $(1, \mathbf{i}, 0,0, \ldots)$ & $\operatorname{det} T_{\alpha, \beta}(n)=F_{n+2}$ & {$[3],[4]$} \\
1 & $(1,-1,0,0, \ldots)$ & $(1,1,0,0, \ldots)$ & $\operatorname{det} T_{\alpha, \beta}(n)=F_{n+2}$ & {$[12]$} \\
3 & $(3,1,0,0, \ldots)$ & $(3,1,0,0, \ldots)$ & $\operatorname{det} T_{\alpha, \beta}(n)=F_{2 n+4}$ & {$[12],[13]$} \\
3 & $(3,-1,0,0, \ldots)$ & $(3,-1,0,0, \ldots)$ & $\operatorname{det} T_{\alpha, \beta}(n)=F_{2 n+4}$ & {$[13]$} \\
1 & $(1, \mathbf{i}, 0,0, \ldots)$ & $(1, \mathbf{i}, 0,0, \ldots)$ & $\operatorname{det}\left(T_{\alpha, \beta}(n)+E_{22}\right)=L_{n+1}$ & {$[3]$} \\
1 & $(1,1,1, \ldots)$ & $(1,-1,0,0, \ldots)$ & $\operatorname{det}\left(T_{\alpha, \beta}(n)+\sum_{i=2}^{n} E_{i i}\right)=F_{2 n+2}$ & {$[3]$} \\
1 & $(1, \mathbf{i}, 0,0, \ldots)$ & $(1, \mathbf{i}, 0,0, \ldots)$ & $\operatorname{det}\left(T_{\alpha, \beta}(n)+2 E_{11}\right)=L_{n+3}$ & {$[2]$} \\
1 & $(1, \Phi, \Phi, \ldots)$ & $(1, \phi, \phi, \ldots)$ & $\operatorname{det} T_{\alpha, \beta}(n)=F_{n+1}$ & {$[7]$} \\
1 & $(0,-\Phi,-\Phi, \ldots)$ & $(0,-\phi,-\phi, \ldots)$ & $\operatorname{det} T_{\alpha, \beta}(n)=F_{n-1}$ & {$[7]$} \\
1 & $\alpha_{i}=\alpha_{i-1}+c$ & $\beta_{i}=\beta_{i-1}-c^{-1}$ & $\operatorname{det} P_{\alpha, \beta}(n)=F_{n+2}$ & {$[11]$} \\
1 & $\alpha_{i}=\alpha_{i-1}+1$ & $\beta_{1}=0, \beta_{i}=\beta_{i+2}$ & $\operatorname{det} A_{\alpha, \beta}(n)=F_{n+1}$ & {$[11]$} \\
1 & $\alpha_{i}=\alpha_{i-1}-1$ & $\beta_{1}=0, \beta_{i}=\beta_{i+2}$ & $\operatorname{det} A_{\alpha, \beta}(n)=F_{n+1}$ & {$[11]$} \\
1 & $\alpha_{i}=\alpha_{i-1}+\mathbf{i}$ & $\beta_{1}=2 \mathbf{i}, \beta_{i}=\beta_{i+2}$ & $\operatorname{det} A_{\alpha, \beta}(n)=L_{n+1}$ & {$[11]$} \\
1 & $\alpha_{i}=\alpha_{i-1}-\mathbf{i}$ & $\beta_{1}=-2 \mathbf{i}, \beta_{i}=\beta_{i+2}$ & $\operatorname{det} A_{\alpha, \beta}(n)=L_{n+1}$ &
\end{tabular}

The purpose of this article is twofold. First, we want to find some new infinite families of integer matrices whose leading principal minors form the sequences $\left(F_{n+1}\right)_{n \geq 0},\left(F_{n}\right)_{n \geq 0},\left(P_{n}\right)_{n \geq 0},\left(J_{n}\right)_{n \geq 0}$ and $\left(L_{n}\right)_{n \geq 0}$. Second, we provide a new proof for Theorem 3.1 in [1].

2. Preliminary results. In this section, we introduce some definitions and several auxiliary results to be used later. We begin with the following definition.

Definition 2.1 (See [15]). Infinite matrices $A=\left[a_{i, j}\right]_{i, j \geq 0}$ and $B=\left[b_{i, j}\right]_{i, j \geq 0}$ are equimodular if the sequences of their leading principal minors agree, i.e.,

$$
\operatorname{det}\left[a_{i, j}\right]_{0 \leq i, j \leq n}=\operatorname{det}\left[b_{i, j}\right]_{0 \leq i, j \leq n} \text { for all } n \geq 0 .
$$

We continue with the following simple observations.

Lemma 2.2 (See [10]). Let $i, j$ be positive integers. Then, we have

$$
\sum_{k=0}^{i-j}(-1)^{k}\left(\begin{array}{c}
i \\
k+j
\end{array}\right)\left(\begin{array}{c}
k+j \\
j
\end{array}\right)=\left\{\begin{array}{lll}
0 & \text { if } & i \neq j \\
1 & \text { if } & i=j .
\end{array}\right.
$$


Lemma 2.3 (See [10]). Let $\varphi$ be an arbitrary sequence. Then, we have $\hat{\tilde{\varphi}}=\check{\hat{\varphi}}=$ $\varphi$.

In [10], we showed that generalized Pascal triangles are strongly related to Toeplitz matrices. Actually, we obtained a factorization of the generalized Pascal triangle $P_{\alpha, \beta}(n)$ associated with the arbitrary sequences $\alpha$ and $\beta$, as a product of the unipotent lower triangular matrix $L(n)$, the Toeplitz matrix $T_{\hat{\alpha}, \hat{\beta}}(n)$ and the unipotent upper triangular matrix $U(n)$ (see [10] and [14]), that is

$$
P_{\alpha, \beta}(n)=L(n) \cdot T_{\hat{\alpha}, \hat{\beta}}(n) \cdot U(n) .
$$

Moreover, we showed that

$$
T_{\alpha, \beta}(n)=L(n)^{-1} \cdot P_{\check{\alpha}, \check{\beta}}(n) \cdot U(n)^{-1} .
$$

In fact, we obtained a relationship between generalized Pascal triangles and Toeplitz matrices. In particular, from the identities (2.1) and (2.2), it follows that

$$
\operatorname{det} P_{\alpha, \beta}(n)=\operatorname{det} T_{\hat{\alpha}, \hat{\beta}}(n) \quad \text { and } \quad \operatorname{det} T_{\alpha, \beta}(n)=\operatorname{det} P_{\check{\alpha}, \check{\beta}}(n) .
$$

Note that, in general, we have

$$
P_{\alpha, \beta}(n)=P_{\beta, \alpha}(n)^{t} \quad \text { and } \quad T_{\alpha, \beta}(n)=T_{\beta, \alpha}(n)^{t} .
$$

Hence, one can immediately obtain the following results:

$$
\operatorname{det} P_{\alpha, \beta}(n)=\operatorname{det} P_{\beta, \alpha}(n) \quad \text { and } \quad \operatorname{det} T_{\alpha, \beta}(n)=\operatorname{det} T_{\beta, \alpha}(n) .
$$

In this section, we first give a factorization of $A_{\alpha, \beta}(n)$ as a product of the unipotent lower triangular matrix and a Toeplitz matrix. The next result is already obtained in $[6,14]$. However, we will include a brief new proof for completeness.

THEOREM 2.4. We have the following factorizations:

$$
A_{\alpha, \beta}(n)=L(n) \cdot T_{\hat{\alpha}, \beta}(n)
$$

and

$$
T_{\alpha, \beta}(n)=L(n)^{-1} \cdot A_{\check{\alpha}, \beta}(n) .
$$

In particular, we have $\operatorname{det} A_{\alpha, \beta}(n)=\operatorname{det} T_{\hat{\alpha}, \beta}(n)$ and $\operatorname{det} T_{\alpha, \beta}(n)=\operatorname{det} A_{\check{\alpha}, \beta}(n)$.

As a matter of fact, Theorem 2.4 gives a connection between 7 -matrices and Toeplitz matrices. For example, we have the following factorizations of $A_{\alpha, \beta}(2)$ and $T_{\alpha, \beta}(2)$ as follows:

$$
\left[\begin{array}{lll}
\gamma & \beta_{1} & \beta_{2} \\
\alpha_{1} & \gamma+\beta_{1} & \beta_{1}+\beta_{2} \\
\alpha_{2} & \alpha_{1}+\gamma+\beta_{1} & \gamma+2 \beta_{1}+\beta_{2}
\end{array}\right]=\left[\begin{array}{lll}
1 & 0 & 0 \\
1 & 1 & 0 \\
1 & 2 & 1
\end{array}\right] \cdot\left[\begin{array}{lll}
\gamma & \beta_{1} & \beta_{2} \\
-\gamma+\alpha_{1} & \gamma & \beta_{1} \\
\gamma-2 \alpha_{1}+\alpha_{2} & -\gamma+\alpha_{1} & \gamma
\end{array}\right]
$$




$$
\left[\begin{array}{lll}
\gamma & \beta_{1} & \beta_{2} \\
\alpha_{1} & \gamma & \beta_{1} \\
\alpha_{2} & \alpha_{1} & \gamma
\end{array}\right]=\left[\begin{array}{ccc}
1 & 0 & 0 \\
-1 & 1 & 0 \\
1 & -2 & 1
\end{array}\right] \cdot\left[\begin{array}{lll}
\gamma & \beta_{1} & \beta_{2} \\
\gamma+\alpha_{1} & \gamma+\beta_{1} & \beta_{1}+\beta_{2} \\
\gamma+2 \alpha_{1}+\alpha_{2} & 2 \gamma+\alpha_{1}+\beta_{1} & \gamma+2 \beta_{1}+\beta_{2}
\end{array}\right]
$$

Proof of Theorem 2.4. We recall that, the 7-matrix $L(n)=\left(L_{i, j}\right)_{0 \leq i, j \leq n}$ is a lower triangular matrix with

$$
L_{i, j}=\left\{\begin{array}{cl}
0 & \text { if } \quad i<j \\
\left(\begin{array}{l}
i \\
j
\end{array}\right) & \text { if } \quad i \geq j
\end{array}\right.
$$

For convenience, we put $A=A_{\alpha, \beta}(n), L=L(n)$ and $T=T_{\hat{\alpha}, \beta}(n)=\left(T_{i, j}\right)_{0 \leq i, j \leq n}$. For the proof of the claimed factorization Eq. (2.5), i.e., $A=L \cdot T$, we compute the $(i, j)$-entry of $L \cdot T$, that is,

$$
(L \cdot T)_{i, j}=\sum_{k=0}^{i} L_{i, k} T_{k, j}
$$

In fact, so as to prove the theorem, we should establish

$$
\begin{aligned}
& \mathrm{R}_{0}(L \cdot T)=\mathrm{R}_{0}(A)=\left(\beta_{0}, \beta_{1}, \ldots, \beta_{n}\right), \\
& \mathrm{C}_{0}(L \cdot T)=\mathrm{C}_{0}(A)=\left(\alpha_{0}, \alpha_{1}, \ldots, \alpha_{n}\right)^{t},
\end{aligned}
$$

and

$$
(L \cdot T)_{i, j}=(L \cdot T)_{i-1, j-1}+(L \cdot T)_{i-1, j}, \quad \text { for } \quad 1 \leq i, j \leq n .
$$

Let us do the required calculations. First, suppose that $i=0$. Then

$$
(L \cdot T)_{0, j}=\sum_{k=0}^{0} L_{0, k} T_{k, j}=L_{0,0} T_{0, j}=\beta_{j}
$$

and so $\mathrm{R}_{0}(L \cdot T)=\mathrm{R}_{0}(A)=\left(\beta_{0}, \beta_{1}, \ldots, \beta_{n}\right)$.

Next, we suppose that $j=0$, and we obtain

$$
\begin{aligned}
(L \cdot T)_{i, 0}=\sum_{k=0}^{i} L_{i, k} T_{k, 0} & =\sum_{k=0}^{i}\left(\begin{array}{c}
i \\
k
\end{array}\right)\left\{\sum_{s=0}^{k}(-1)^{s+k}\left(\begin{array}{c}
k \\
s
\end{array}\right) \alpha_{s}\right\} \\
& =\sum_{r=0}^{i} \alpha_{r}\left\{\sum_{s=0}^{i-r}(-1)^{s}\left(\begin{array}{c}
i \\
r+s
\end{array}\right)\left(\begin{array}{c}
r+s \\
r
\end{array}\right)\right\} \\
& =\alpha_{i}, \quad \text { (by Lemma 2.2) }
\end{aligned}
$$


which implies that $\mathrm{C}_{0}(L \cdot T)=\mathrm{C}_{0}(A)=\left(\alpha_{0}, \alpha_{1}, \ldots, \alpha_{n}\right)^{t}$.

Finally, we must establish Eq. (2.7). At the moment, let us assume that $1 \leq$ $i, j \leq n$. Note that the entries of $L$ satisfy in the following recurrence

$$
L_{i, j}=L_{i-1, j-1}+L_{i-1, j}, \quad 1 \leq i, j \leq n .
$$

Now, we obtain that

$$
\begin{aligned}
(L \cdot T)_{i, j}= & \sum_{k=0}^{i} L_{i, k} T_{k, j} \\
= & L_{i, 0} T_{0, j}+\sum_{k=1}^{i} L_{i, k} T_{k, j} \\
= & \left.L_{i, 0} T_{0, j}+\sum_{k=1}^{i}\left(L_{i-1, k-1}+L_{i-1, k}\right) T_{k, j} \quad \text { (by Eq. (2.8) }\right) \\
= & L_{i, 0} T_{0, j}+\sum_{k=1}^{i} L_{i-1, k-1} T_{k, j}+\sum_{k=1}^{i} L_{i-1, k} T_{k, j} \\
= & L_{i, 0} T_{0, j}+\sum_{k=1}^{i} L_{i-1, k-1} T_{k-1, j-1}+\sum_{k=0}^{i} L_{i-1, k} T_{k, j}-L_{i-1,0} T_{0, j} \\
& \left(\text { Notice that } T_{k, j}=T_{k-1, j-1}\right) \\
= & \left(L_{i, 0}-L_{i-1,0}\right) T_{0, j}+\sum_{k=0}^{i} L_{i-1, k} T_{k, j-1}+\sum_{k=0}^{i} L_{i-1, k} T_{k, j} \\
& \left(\text { It should be noticed that } L_{i-1, i}=0\right) \\
= & (L \cdot T)_{i-1, j-1}+(L \cdot T)_{i-1, j}, \quad\left(\text { note that } L_{i, 0}=L_{i-1,0}=1\right)
\end{aligned}
$$

which is Eq. (2.7).

To prove of Eq. (2.6), we observe that

$$
\begin{aligned}
L(n)^{-1} \cdot A_{\check{\alpha}, \beta}(n) & \left.=L(n)^{-1} \cdot L(n) \cdot T_{\tilde{\alpha}, \beta}(n) \quad \text { (by Eq. }(2.5)\right) \\
& \left.=T_{\alpha, \beta}(n) . \quad \text { (by Lemma } 2.3\right)
\end{aligned}
$$

Our proof is thus complete.

As a consequence, we have the following result.

Corollary 2.5. Let $\alpha$ and $\beta$ be two sequences starting with a common first term. Then, there holds

$$
\operatorname{det} A_{\check{\alpha}, \beta}(n)=\operatorname{det} A_{\check{\beta}, \alpha}(n) .
$$

Proof. By Theorem 2.4, we have

$$
\operatorname{det} A_{\check{\alpha}, \beta}(n)=\operatorname{det} T_{\alpha, \beta}(n) \quad \text { and } \quad \operatorname{det} A_{\breve{\beta}, \alpha}(n)=\operatorname{det} T_{\beta, \alpha}(n) .
$$

Now, the conclusion follows immediately from Eq. (2.4).

We can summarize the above results in the following corollary. 
Corollary 2.6. Let $\alpha$ and $\beta$ be two sequences starting with a common first term. Then, the following matrices

$$
T_{\alpha, \beta}(\infty), \quad T_{\beta, \alpha}(\infty), \quad P_{\check{\alpha}, \check{\beta}}(\infty), \quad P_{\breve{\beta}, \check{\alpha}}(\infty), \quad A_{\check{\alpha}, \beta}(\infty) \text { and } A_{\breve{\beta}, \alpha}(\infty),
$$

are equimodular.

We conclude this section with the following simple observation to be used in the next section.

Lemma 2.7. Let $\alpha$ be an arbitrary sequence. Then, it holds that

$$
\sum_{i=0}^{n-1} \check{\alpha}_{i}=\sum_{j=0}^{n-1}\left(\begin{array}{c}
n \\
j+1
\end{array}\right) \alpha_{j}
$$

Proof. Easy computations show that

$$
\sum_{i=0}^{n-1} \check{\alpha}_{i}=\sum_{i=0}^{n-1} \sum_{k=0}^{i}\left(\begin{array}{l}
i \\
k
\end{array}\right) \alpha_{k}=\sum_{j=0}^{n-1}\left(\sum_{r=0}^{n-1}\left(\begin{array}{l}
r \\
j
\end{array}\right)\right) \alpha_{j}=\sum_{j=0}^{n-1}\left(\begin{array}{c}
n \\
j+1
\end{array}\right) \alpha_{j}
$$

as desired.

3. Main results. As we mentioned in the Introduction, we are going to obtain some integer matrices whose leading principal minors form the Fibonacci, Lucas, Pell and Jacobsthal sequences. We start with the following definition.

Definition 3.1. A sequence $\phi=\left(\phi_{i}\right)_{i \geq 0}$ satisfies a homogeneous linear recurrence relation of order $s$ (with constant coefficients), if there exist constants $C_{1}, C_{2}, \ldots, C_{s}$, with $C_{s} \neq 0$, such that

$$
\phi_{i}=\sum_{r=1}^{s} C_{r} \phi_{i-r}, \quad \text { for all } i \geq s .
$$

Such a sequence is called $s$-recurrent.

We recall that, generally, the binomial transforms preserve the vector space of sequences satisfying linear recurrence relations and also they preserve the set of such sequences satisfying linear recurrence relations of given degree. We illustrate this with the following lemma.

LEMMA 3.2. Let $\alpha=\left(\alpha_{i}\right)_{i \geq 0}$ be a sequence satisfying a homogeneous linear recurrence relation of order 2 :

$$
\alpha_{i}=C_{1} \alpha_{i-1}+C_{2} \alpha_{i-2} \quad \text { for all } i \geq 2
$$


Then, we have

(a) The sequence $\check{\alpha}=\left(\check{\alpha}_{i}\right)_{i \geq 0}$ satisfies a homogeneous linear recurrence relation with $\check{\alpha}_{0}=\alpha_{0}, \check{\alpha}_{1}=\alpha_{0}+\alpha_{1}$, and

$$
\check{\alpha}_{i}=\left(C_{1}+2\right) \check{\alpha}_{i-1}+\left(C_{2}-C_{1}-1\right) \check{\alpha}_{i-2} \text { for all } i \geq 2 \text {. }
$$

(b) The sequence $\hat{\alpha}=\left(\hat{\alpha}_{i}\right)_{i \geq 0}$ satisfies a homogeneous linear recurrence relation with $\hat{\alpha}_{0}=\alpha_{0}, \hat{\alpha}_{1}=-\alpha_{0}+\alpha_{1}$, and

$$
\hat{\alpha}_{i}=\left(C_{1}-2\right) \hat{\alpha}_{i-1}+\left(C_{2}+C_{1}-1\right) \hat{\alpha}_{i-2} \quad \text { for all } i \geq 2
$$

Proof. (a) Evidently $\check{\alpha}_{0}=\alpha_{0}$ and $\check{\alpha}_{1}=\alpha_{0}+\alpha_{1}$. To prove Eq. (3.2), we use strong induction on $i$. It is easy to verify the result if $i=2$. Suppose that the result is true for all $i<n$. We put $S:=\left(C_{1}+2\right) \check{\alpha}_{n-1}+\left(C_{2}-C_{1}-1\right) \check{\alpha}_{n-2}$. We have to prove that $S=\check{\alpha}_{n}$. To do this, we notice that

$$
\begin{aligned}
S & =\left(C_{1}+2\right) \check{\alpha}_{n-1}+\left(C_{2}-C_{1}-1\right) \check{\alpha}_{n-2} \\
\check{\alpha}_{n-1} & =\left(C_{1}+2\right) \check{\alpha}_{n-2}+\left(C_{2}-C_{1}-1\right) \check{\alpha}_{n-3} \\
\check{\alpha}_{n-2} & =\left(C_{1}+2\right) \check{\alpha}_{n-3}+\left(C_{2}-C_{1}-1\right) \check{\alpha}_{n-4} \\
\check{\alpha}_{n-3} & =\left(C_{1}+2\right) \check{\alpha}_{n-4}+\left(C_{2}-C_{1}-1\right) \check{\alpha}_{n-5} \\
& \vdots \\
\check{\alpha}_{2} & =\left(C_{1}+2\right) \check{\alpha}_{1}+\left(C_{2}-C_{1}-1\right) \check{\alpha}_{0} .
\end{aligned}
$$

Adding both sides and simplifying, we obtain

$$
S+\sum_{i=2}^{n-1} \check{\alpha}_{i}=\left(C_{1}+2\right) \check{\alpha}_{n-1}+\left(C_{2}+1\right) \sum_{i=1}^{n-2} \check{\alpha}_{i}+\left(C_{2}-C_{1}-1\right) \check{\alpha}_{0}
$$

or equivalently

$$
S+\check{\alpha}_{n-1}=\left(C_{1}+2\right) \check{\alpha}_{n-1}+C_{2} \sum_{i=1}^{n-2} \check{\alpha}_{i}+\check{\alpha}_{1}+\left(C_{2}-C_{1}-1\right) \check{\alpha}_{0}
$$


After more simplification this leads to

$$
\begin{aligned}
S & =\check{\alpha}_{n-1}+C_{1} \check{\alpha}_{n-1}+C_{2}\left(\sum_{i=0}^{n-2} \check{\alpha}_{i}-\check{\alpha}_{0}\right)+\alpha_{1}+\left(C_{2}-C_{1}\right) \check{\alpha}_{0} \\
& =\check{\alpha}_{n-1}+\sum_{i=0}^{n-1}\left(\begin{array}{c}
n-1 \\
i
\end{array}\right) C_{1} \alpha_{i}+C_{2}\left(\sum_{i=0}^{n-2}\left(\begin{array}{c}
n-1 \\
i+1
\end{array}\right) \alpha_{i}-\alpha_{0}\right)+\alpha_{1}+\left(C_{2}-C_{1}\right) \check{\alpha}_{0}
\end{aligned}
$$

(by definition of $\check{\alpha}_{n-1}$ and Lemma 2.7)

$=\check{\alpha}_{n-1}+\sum_{i=1}^{n-1}\left(\begin{array}{c}n-1 \\ i\end{array}\right)\left(\alpha_{i+1}-C_{2} \alpha_{i-1}\right)+C_{2} \sum_{i=0}^{n-2}\left(\begin{array}{c}n-1 \\ i+1\end{array}\right) \alpha_{i}+\alpha_{1} \quad$ (by Eq. (3.1))

$=\check{\alpha}_{n-1}+\sum_{i=0}^{n-1}\left(\begin{array}{c}n-1 \\ i\end{array}\right) \alpha_{i+1}$

$=\sum_{i=0}^{n-1}\left(\begin{array}{c}n-1 \\ i\end{array}\right) \alpha_{i}+\sum_{i=1}^{n}\left(\begin{array}{c}n-1 \\ i-1\end{array}\right) \alpha_{i}$

$=\alpha_{0}+\sum_{i=1}^{n-1}\left[\left(\begin{array}{c}n-1 \\ i\end{array}\right)+\left(\begin{array}{c}n-1 \\ i-1\end{array}\right)\right] \alpha_{i}+\alpha_{n}$

$=\alpha_{0}+\sum_{i=1}^{n-1}\left(\begin{array}{c}n \\ i\end{array}\right) \alpha_{i}+\alpha_{n} \quad$ (by Pascal's rule)

$=\sum_{i=0}^{n}\left(\begin{array}{c}n \\ i\end{array}\right) \alpha_{i}=\check{\alpha}_{n}$,

which completes the proof of $(a)$.

(b) The proof of this part is similar to that of part $(a)$ and, therefore, omitted.

Let us consider Toeplitz matrices in which the first row and column are linear recursion sequences of order 2 . Indeed, for Toeplitz matrices associated with two linear recursion sequences of order 2 , we will prove that the sequence of their leading principal minors satisfies such a recurrence of order 2 as well. More precisely, let $\alpha=\left(\alpha_{i}\right)_{i \geq 0}$ and $\beta=\left(\beta_{i}\right)_{i \geq 0}$ be two sequences satisfying $\alpha_{0}=\beta_{0}=\gamma$ and linear recursion

$$
\alpha_{i}=A_{1} \alpha_{i-1}+A_{2} \alpha_{i-2} \quad \text { and } \quad \beta_{i}=B_{1} \beta_{i-1}+B_{2} \beta_{i-2} \quad \text { for all } i \geq 2,
$$

of order 2 . We set $d_{n}=\operatorname{det} T_{\alpha, \beta}(n)$, for $n \geq 0$, and introduce the following constants:

$$
D_{1}=\gamma\left(1+A_{1} B_{1}-A_{2} B_{2}\right)-\alpha_{1} B_{1}-\beta_{1} A_{1},
$$

and

$$
D_{2}=-\left(\alpha_{1}-A_{1} \gamma-A_{2} \beta_{1}\right)\left(\beta_{1}-B_{2} \alpha_{1}-B_{1} \gamma\right)
$$

Then, we have the following result. 
THEOREM 3.3. The sequence $d_{n}, n \geq 0$, defined as above, satisfies the following equalities

$$
\begin{aligned}
& d_{0}=\gamma \\
& d_{1}=\gamma^{2}-\alpha_{1} \beta_{1}, \\
& d_{n}=D_{1} d_{n-1}+D_{2} d_{n-2} \quad \text { for all } n \geq 2 .
\end{aligned}
$$

Proof. The assertions concerning $d_{0}$ and $d_{1}$ are obvious. Hence, we may assume that $n \geq 2$. Now, we introduce the lower and upper triangular square matrices

$$
T_{A}(n)=\left[\begin{array}{ccc}
1 & 0 & 0 \\
-A_{1} & 1 & 0 \\
-A_{2} & 0 & 1
\end{array}\right] \oplus I_{n-2} \text { and } T_{B}(n)=\left[\begin{array}{ccc}
1 & -B_{1} & -B_{2} \\
0 & 1 & 0 \\
0 & 0 & 1
\end{array}\right] \oplus I_{n-2}
$$

where $I_{m}$ is the identity matrix of order $m$, and we set

$$
\widetilde{T}(n)=T_{B}(n) \cdot T_{\alpha, \beta}(n) \cdot T_{A}(n) .
$$

One has

$$
\widetilde{T}(n)=\left[\begin{array}{ccccccc}
\widetilde{T}_{0,0} & \widetilde{T}_{0,1} & 0 & 0 & 0 & \ldots & 0 \\
\widetilde{T}_{1,0} & \gamma & \beta_{1} & \beta_{2} & \beta_{3} & \ldots & \beta_{n-1} \\
0 & \alpha_{1} & \gamma & \beta_{1} & \beta_{2} & \ddots & \vdots \\
0 & \alpha_{2} & \alpha_{1} & \gamma & \beta_{1} & \ddots & \beta_{3} \\
0 & \alpha_{3} & \alpha_{2} & \alpha_{1} & \gamma & \ddots & \beta_{2} \\
\vdots & \vdots & \ddots & \ddots & \ddots & \ddots & \beta_{1} \\
0 & \alpha_{n-1} & \ldots & \alpha_{3} & \alpha_{2} & \alpha_{1} & \gamma
\end{array}\right]
$$

where

$$
\begin{aligned}
& \widetilde{T}_{0,0}=\gamma\left(1+A_{1} B_{1}-A_{2} B_{2}\right)-\alpha_{1} B_{1}-\beta_{1} A_{1} \\
& \widetilde{T}_{1,0}=\alpha_{1}-A_{1} \gamma-A_{2} \beta_{1} \\
& \widetilde{T}_{0,1}=\beta_{1}-B_{2} \alpha_{1}-B_{1} \gamma .
\end{aligned}
$$

Developing the determinant $d_{n}=\operatorname{det} \widetilde{T}(n)$ along the first column of $\widetilde{T}(n)$ one obtains

$$
d_{n}=D_{1} d_{n-1}+D_{2} d_{n-2},
$$

as required. $\square$

As consequences of Theorem 3.3, we have the following corollaries. 
Corollary 3.4. Let $A_{1}, A_{2}, B_{1}$ and $B_{2}$ be integers with $A_{2}, B_{2} \neq 0$. Let $\alpha=\left(\alpha_{i}\right)_{i \geq 0}$ and $\beta=\left(\beta_{i}\right)_{i \geq 0}$ be two integer sequences satisfying $\alpha_{0}=\beta_{0}=\gamma$ and linear recurrences

$$
\alpha_{i}=A_{1} \alpha_{i-1}+A_{2} \alpha_{i-2} \quad \text { and } \quad \beta_{i}=B_{1} \beta_{i-1}+B_{2} \beta_{i-2} \quad \text { for all } i \geq 2,
$$

of order 2. Then it holds

(a) For any nonnegative integer $n$, we have $\operatorname{det} T_{\alpha, \beta}(n)=F_{n+1}$ if and only if $\gamma, \alpha_{1}, \beta_{1}, A_{1}, A_{2}, B_{1}$ and $B_{2}$ satisfy one of the following conditions, where $c$ is a constant.

\begin{tabular}{|c|ccccccc|}
\hline & $\gamma$ & $\alpha_{1}$ & $\beta_{1}$ & $A_{1}$ & $A_{2}$ & $B_{1}$ & $B_{2}$ \\
\hline$(\mathrm{F} .1)$ & 1 & 0 & $c+1$ & $-c$ & 1 & $c$ & $c$ \\
$(\mathrm{~F} .2)$ & 1 & 0 & $c-1$ & $c$ & -1 & $c-2$ & $c$ \\
$(\mathrm{~F} .3)$ & 1 & 0 & $c-1$ & $-c$ & 1 & $c$ & $-c$ \\
$(\mathrm{~F} .4)$ & 1 & 0 & $1-c$ & $-c$ & -1 & $2-c$ & $c$ \\
$(\mathrm{~F} .5)$ & 1 & $c+1$ & 0 & $c$ & $c$ & $-c$ & 1 \\
$(\mathrm{~F} .6)$ & 1 & $c-1$ & 0 & $c-2$ & $c$ & $c$ & -1 \\
$(\mathrm{~F} .7)$ & 1 & $c-1$ & 0 & $c$ & $-c$ & $-c$ & 1 \\
$(\mathrm{~F} .8)$ & 1 & $1-c$ & 0 & $2-c$ & $c$ & $-c$ & -1 \\
\hline
\end{tabular}

(b) For any nonnegative integer $n$, we have $\operatorname{det} A_{\alpha, \beta}(n)=F_{n+1}$ if and only if $\gamma, \alpha_{1}, \beta_{1}, A_{1}, A_{2}, B_{1}$ and $B_{2}$ satisfy one of the following conditions, where $c$ is a constant.

\begin{tabular}{|c|ccccccc|}
\hline & $\gamma$ & $\alpha_{1}$ & $\beta_{1}$ & $A_{1}$ & $A_{2}$ & $B_{1}$ & $B_{2}$ \\
\hline$\left(\mathrm{F} .1^{\prime}\right)$ & 1 & 1 & $c+1$ & $2-c$ & $c$ & $c$ & $c$ \\
$\left(\mathrm{~F} .2^{\prime}\right)$ & 1 & 1 & $c-1$ & $c+2$ & $-(c+2)$ & $c-2$ & $c$ \\
$\left(\mathrm{~F} .3^{\prime}\right)$ & 1 & 1 & $c-1$ & $2-c$ & $c$ & $c$ & $-c$ \\
$\left(\mathrm{~F} .4^{\prime}\right)$ & 1 & 1 & $1-c$ & $2-c$ & $c-2$ & $2-c$ & $c$ \\
$\left(\mathrm{~F} .5^{\prime}\right)$ & 1 & $c+2$ & 0 & $2+c$ & -1 & $-c$ & 1 \\
$\left(\mathrm{~F} .6^{\prime}\right)$ & 1 & $c$ & 0 & $c$ & 1 & $c$ & -1 \\
$\left(\mathrm{~F} .7^{\prime}\right)$ & 1 & $c$ & 0 & $c+2$ & $-(2 c+1)$ & $-c$ & 1 \\
$\left(\mathrm{~F} .8^{\prime}\right)$ & 1 & $2-c$ & 0 & $4-c$ & $2 c-3$ & $-c$ & -1 \\
\hline
\end{tabular}

(c) For any nonnegative integer $n$, we have $\operatorname{det} P_{\alpha, \beta}(n)=F_{n+1}$, if and only if $\gamma, \alpha_{1}, \beta_{1}, A_{1}, A_{2}, B_{1}$ and $B_{2}$ satisfy one of the following conditions, where $c$ is a constant. 


\begin{tabular}{|c|ccccccc|}
\hline & $\gamma$ & $\alpha_{1}$ & $\beta_{1}$ & $A_{1}$ & $A_{2}$ & $B_{1}$ & $B_{2}$ \\
\hline$\left(\mathrm{F} .1^{\prime \prime}\right)$ & 1 & 1 & $c+2$ & $2-c$ & $c$ & $2+c$ & -1 \\
$\left(\mathrm{~F} .2^{\prime \prime}\right)$ & 1 & 1 & $c$ & $c+2$ & $-(c+2)$ & $c$ & 1 \\
$\left(\mathrm{~F} .3^{\prime \prime}\right)$ & 1 & 1 & $c$ & $2-c$ & $c$ & $c+2$ & $-(2 c+1)$ \\
$\left(\mathrm{F} .4^{\prime \prime}\right)$ & 1 & 1 & $2-c$ & $2-c$ & $c-2$ & $4-c$ & $2 c-3$ \\
$\left(\mathrm{~F} .5^{\prime \prime}\right)$ & 1 & $c+2$ & 1 & $2+c$ & -1 & $2-c$ & $c$ \\
$\left(\mathrm{~F} .6^{\prime \prime}\right)$ & 1 & $c$ & 1 & $c$ & 1 & $c+2$ & $-(c+2)$ \\
$\left(\mathrm{F} .7^{\prime \prime}\right)$ & 1 & $c$ & 1 & $c+2$ & $-(2 c+1)$ & $2-c$ & $c$ \\
$\left(\mathrm{~F} .8^{\prime \prime}\right)$ & 1 & $2-c$ & 1 & $4-c$ & $2 c-3$ & $2-c$ & $c-2$ \\
\hline
\end{tabular}

REMARK 3.5. Although we have restricted ourselves to integral values in Corollary 3.4, the necessary part holds for arbitrary values of $c$. It is worth mentioning that the Toeplitz matrix constructed by condition (F.1) (resp., (F.2), (F.3) and (F.4)) is the transpose of the Toeplitz matrix constructed by condition (F.5) (resp., (F.6), (F.7) and (F.8)). In addition, by Corollary 3.4, there exist some new infinite families of Toeplitz matrices, 7-matrices and generalized Pascal triangles whose leading principal minors form the Fibonacci sequence.

Proof of Corollary 3.4. (a) The sufficiency is clear. To prove the necessity, suppose that

$$
d_{n}=\operatorname{det} T_{\alpha, \beta}(n)=F_{n+1} .
$$

By Theorem 3.3, it follows that $d_{0}=\gamma, d_{1}=\gamma^{2}-\alpha_{1} \beta_{1}$, and for all $n \geq 2$,

$$
d_{n}=D_{1} d_{n-1}+D_{2} d_{n-2},
$$

where

$$
D_{1}=\gamma\left(1+A_{1} B_{1}-A_{2} B_{2}\right)-\alpha_{1} B_{1}-\beta_{1} A_{1},
$$

and

$$
D_{2}=-\left(\alpha_{1}-A_{1} \gamma-A_{2} \beta_{1}\right)\left(\beta_{1}-B_{2} \alpha_{1}-B_{1} \gamma\right)
$$

Note that, by our assumption we have $D_{1}=D_{2}=1$. Since $d_{0}=F_{1}$, we conclude that $\gamma=1$. Moreover, from $d_{1}=F_{2}$, it follows that $\gamma^{2}-\alpha_{1} \beta_{1}=1$, and since $\gamma=1$, 
we deduce that $\alpha_{1} \beta_{1}=0$, which implies that $\alpha_{1}=0$ or $\beta_{1}=0$. Since the proofs of both cases are similar, only the proof for $\alpha_{1}=0$ is presented.

We assume that $\alpha_{1}=0$. If this is substituted in Eqs. (3.3) and (3.4), then we obtain

$$
A_{1} B_{1}-A_{2} B_{2}-\beta_{1} A_{1}=0
$$

and

$$
\left(A_{1}+A_{2} \beta_{1}\right)\left(\beta_{1}-B_{1}\right)=1 .
$$

Solving Eq. (3.6) in $\mathbb{Z}$, we obtain that

$$
\left(A_{1}+A_{2} \beta_{1}, \beta_{1}-B_{1}\right) \in\{(1,1),(-1,-1)\} .
$$

We now consider two cases separately.

Case 1. $A_{1}+A_{2} \beta_{1}=1$ and $\beta_{1}-B_{1}=1$.

In this case, we have $\beta_{1}=B_{1}+1$. After having substituted this in Eq. (3.5), we conclude that $A_{1}=-A_{2} B_{2}$. Now, if we substitute $-A_{2} B_{2}$ with $A_{1}$ in $A_{1}+A_{2} \beta_{1}=1$, we obtain $A_{2}\left(\beta_{1}-B_{2}\right)=1$. This yields that $\left(A_{2}, \beta_{1}-B_{2}\right)=(1,1)$ or $\left(A_{2}, \beta_{1}-\right.$ $\left.B_{2}\right)=(-1,-1)$. If $\left(A_{2}, \beta_{1}-B_{2}\right)=(1,1)$, then $B_{1}=B_{2}=-A_{1}(:=c$ say $)$, and if $\left(A_{2}, \beta_{1}-B_{2}\right)=(-1,-1)$, then $B_{1}-2=B_{2}=A_{1}(:=c$ say $)$.

Case 2. $A_{1}+A_{2} \beta_{1}=-1$ and $\beta_{1}-B_{1}=-1$.

The proof is quite similar to the proof in Case 1, so we avoid here full explanation of all details. In fact, we obtain $A_{2}=1$ and $B_{1}=-B_{2}=-A_{1}(:=c$ say $)$ or $A_{2}=-1$ and $2-B_{1}=B_{2}=-A_{1}(:=c$ say $)$.

(b) This follows directly from part (a), Theorem 2.4, and Lemma $3.2(a)$.

(c) This follows from part (a), Lemma $3.2(a)$ and Eq. (2.3).

As a special case of Corollary 3.4, we see that Fibonacci numbers provide the following exotic example:

ExAmple 3.6. We have

$$
\operatorname{det} A_{\left(F_{1}, F_{2}, F_{3}, \ldots\right),\left(F_{2}, F_{3}, F_{4}, \ldots\right)}(n)=F_{n+1} .
$$

In fact, this example follows from Corollary $3.4(b)$, part $\left(\mathrm{F} .1^{\prime}\right)$, if we substitute 1 with c.

Corollary 3.7. Let $A_{1}, A_{2}, B_{1}$ and $B_{2}$ be integers. Let $\alpha=\left(\alpha_{i}\right)_{i \geq 0}$ and $\beta=\left(\beta_{i}\right)_{i \geq 0}$ be two integer sequences satisfy $\alpha_{0}=\beta_{0}=\gamma$ and linear recurrences

$$
\alpha_{i}=A_{1} \alpha_{i-1}+A_{2} \alpha_{i-2} \quad \text { and } \quad \beta_{i}=B_{1} \beta_{i-1}+B_{2} \beta_{i-2} \quad \text { for all } i \geq 2 .
$$


Then it holds

(a) For any nonnegative integer $n$, $\operatorname{det} T_{\alpha, \beta}(n)=L_{n}$ if and only if $\gamma, \alpha_{1}, \beta_{1}, A_{1}$, $A_{2}, B_{1}$ and $B_{2}$ satisfy one of the following conditions.

\begin{tabular}{|c|c|c|c|c|c|c|c|c|c|c|c|c|c|c|c|}
\hline & $\gamma$ & $\alpha_{1}$ & $\beta_{1}$ & $A_{1}$ & $A_{2}$ & $B_{1}$ & $B_{2}$ & & $\gamma$ & $\alpha_{1}$ & $\beta_{1}$ & $A_{1}$ & $A_{2}$ & $B_{1}$ & $B_{2}$ \\
\hline (L.1) & 2 & 3 & 1 & 0 & 2 & -5 & 4 & (L.17) & 2 & 1 & 3 & -5 & 4 & 0 & 2 \\
\hline (L.2) & 2 & 3 & 1 & 4 & -4 & -9 & 6 & (L.18) & 2 & 1 & 3 & -9 & 6 & 4 & -4 \\
\hline (L.3) & 2 & 3 & 1 & -1 & 4 & 10 & -6 & (L.19) & 2 & 1 & 3 & 10 & -6 & -1 & 4 \\
\hline (L.4) & 2 & 3 & 1 & 3 & -2 & 6 & -4 & (L.20) & 2 & 1 & 3 & 6 & -4 & 3 & -2 \\
\hline (L.5) & 2 & 3 & 1 & 6 & -8 & -3 & 2 & (L.21) & 2 & 1 & 3 & -3 & 2 & 6 & -8 \\
\hline (L.6) & 2 & 3 & 1 & 2 & -2 & 1 & 0 & (L.22) & 2 & 1 & 3 & 1 & 0 & 2 & -2 \\
\hline (L.7) & 2 & 3 & 1 & -3 & 8 & 4 & -2 & (L.23) & 2 & 1 & 3 & 4 & -2 & -3 & 8 \\
\hline (L.8) & 2 & 3 & 1 & 1 & 2 & 0 & 0 & (L.24) & 2 & 1 & 3 & 0 & 0 & 1 & 2 \\
\hline (L.9) & 2 & -3 & -1 & -2 & -2 & -1 & 0 & (L.25) & 2 & -1 & -3 & -1 & 0 & -2 & -2 \\
\hline (L.10) & 2 & -3 & -1 & -6 & -8 & 3 & 2 & (L.26) & 2 & -1 & -3 & 3 & 2 & -6 & -8 \\
\hline (L.11) & 2 & -3 & -1 & 0 & 2 & 5 & 4 & (L.27) & 2 & -1 & -3 & 5 & 4 & 0 & 2 \\
\hline (L.12) & 2 & -3 & -1 & -4 & -4 & 9 & 6 & (L.28) & 2 & -1 & -3 & 9 & 6 & -4 & -4 \\
\hline (L.13) & 2 & -3 & -1 & 1 & 4 & -10 & -6 & (L.29) & 2 & -1 & -3 & -10 & -6 & 1 & 4 \\
\hline (L.14) & 2 & -3 & -1 & -3 & -2 & -6 & -4 & (L.30) & 2 & -1 & -3 & -6 & -4 & -3 & -2 \\
\hline (L.15) & 2 & -3 & -1 & 3 & 8 & -4 & -2 & (L.31) & 2 & -1 & -3 & -4 & -2 & 3 & 8 \\
\hline (L.16) & 2 & -3 & -1 & -1 & 2 & 0 & 0 & (L.32) & 2 & -1 & -3 & 0 & 0 & -1 & 2 \\
\hline
\end{tabular}

(b) For any nonnegative integer $n, \operatorname{det} A_{\alpha, \beta}(n)=L_{n}$ if and only if $\gamma, \alpha_{1}, \beta_{1}, A_{1}$, $A_{2}, B_{1}$ and $B_{2}$ satisfy one of the following conditions.

\begin{tabular}{|c|c|c|c|c|c|c|c|c|c|c|c|c|c|c|c|}
\hline & $\gamma$ & $\alpha_{1}$ & $\beta_{1}$ & $A_{1}$ & $A_{2}$ & $B_{1}$ & $B_{2}$ & & $\gamma$ & $\alpha_{1}$ & $\beta_{1}$ & $A_{1}$ & $A_{2}$ & $B_{1}$ & $B_{2}$ \\
\hline$\left(\mathrm{L} .1^{\prime}\right)$ & 2 & 5 & 1 & 2 & 1 & -5 & 4 & $\left(\mathrm{~L} .17^{\prime}\right)$ & 2 & 3 & 3 & -3 & 8 & 0 & 2 \\
\hline$\left(\mathrm{L} .2^{\prime}\right)$ & 2 & 5 & 1 & 6 & -9 & -9 & 6 & (L.18') & 2 & 3 & 3 & -7 & 14 & 4 & -4 \\
\hline$\left(\mathrm{L} .3^{\prime}\right)$ & 2 & 5 & 1 & 1 & 4 & 10 & -6 & (L.19') & 2 & 3 & 3 & 12 & -17 & -1 & 4 \\
\hline$\left(\mathrm{L} .4^{\prime}\right)$ & 2 & 5 & 1 & 5 & -6 & 6 & -4 & $\left(\mathrm{~L} .20^{\prime}\right)$ & 2 & 3 & 3 & 8 & -11 & 3 & -2 \\
\hline$\left(\mathrm{L} .5^{\prime}\right)$ & 2 & 5 & 1 & 8 & -15 & -3 & 2 & $\left(\mathrm{~L} .21^{\prime}\right)$ & 2 & 3 & 3 & -1 & 4 & 6 & -8 \\
\hline$\left(\right.$ L. $\left.6^{\prime}\right)$ & 2 & 5 & 1 & 4 & -5 & 1 & 0 & $\left(\mathrm{~L} .22^{\prime}\right)$ & 2 & 3 & 3 & 3 & -2 & 2 & -2 \\
\hline$\left(\mathrm{L} .7^{\prime}\right)$ & 2 & 5 & 1 & -1 & 10 & 4 & -2 & (L.23') & 2 & 3 & 3 & 6 & -7 & -3 & 8 \\
\hline$\left(\mathrm{L} .8^{\prime}\right)$ & 2 & 5 & 1 & 3 & 0 & 0 & 0 & $\left(\mathrm{~L} .24^{\prime}\right)$ & 2 & 3 & 3 & 2 & -1 & 1 & 2 \\
\hline$\left(\mathrm{L} .9^{\prime}\right)$ & 2 & -1 & -1 & 0 & -1 & -1 & 0 & $\left(\mathrm{~L} .25^{\prime}\right)$ & 2 & 1 & -3 & 1 & 0 & -2 & -2 \\
\hline (L.10') & 2 & -1 & -1 & -4 & -3 & 3 & 2 & $\left(\mathrm{~L} .26^{\prime}\right)$ & 2 & 1 & -3 & 5 & -2 & -6 & -8 \\
\hline (L.11') & 2 & -1 & -1 & 2 & 1 & 5 & 4 & $\left(\mathrm{~L} .27^{\prime}\right)$ & 2 & 1 & -3 & 7 & -2 & 0 & 2 \\
\hline (L.12') & 2 & -1 & -1 & -2 & -1 & 9 & 6 & (L.28') & 2 & 1 & -3 & 11 & -4 & -4 & -4 \\
\hline$\left(\mathrm{L} .13^{\prime}\right)$ & 2 & -1 & -1 & 3 & 2 & -10 & -6 & $\left(\mathrm{~L} .29^{\prime}\right)$ & 2 & 1 & -3 & -8 & 3 & 1 & 4 \\
\hline (L.14') & 2 & -1 & -1 & -1 & 0 & -6 & -4 & (L.30') & 2 & 1 & -3 & -4 & 1 & -3 & -2 \\
\hline (L.15') & 2 & -1 & -1 & 5 & 4 & -4 & -2 & $\left(\mathrm{~L} .31^{\prime}\right)$ & 2 & 1 & -3 & -2 & 1 & 3 & 8 \\
\hline$\left(\mathrm{L} .16^{\prime}\right)$ & 2 & -1 & -1 & 1 & 2 & 0 & 0 & (L.32') & 2 & 1 & -3 & 2 & -1 & -1 & 2 \\
\hline
\end{tabular}


(c) For any nonnegative integer $n$, $\operatorname{det} P_{\alpha, \beta}(n)=L_{n}$ if and only if $\gamma, \alpha_{1}, \beta_{1}, A_{1}$, $A_{2}, B_{1}$ and $B_{2}$ satisfy one of the following conditions.

\begin{tabular}{|c|rrrrrrr||l|lllllrr|}
\hline & $\gamma$ & $\alpha_{1}$ & $\beta_{1}$ & $A_{1}$ & $A_{2}$ & $B_{1}$ & $B_{2}$ & & $\gamma$ & $\alpha_{1}$ & $\beta_{1}$ & $A_{1}$ & $A_{2}$ & $B_{1}$ & $B_{2}$ \\
\hline$\left(\mathrm{L} .1^{\prime \prime}\right)$ & 2 & 5 & 3 & 2 & 1 & -3 & 8 & $\left(\mathrm{~L} .17^{\prime \prime}\right)$ & 2 & 3 & 5 & -3 & 8 & 2 & 1 \\
$\left(\mathrm{~L} .2^{\prime \prime}\right)$ & 2 & 5 & 3 & 6 & -9 & -7 & 14 & $\left(\mathrm{~L} .18^{\prime \prime}\right)$ & 2 & 3 & 5 & -7 & 14 & 6 & -9 \\
$\left(\mathrm{~L} .3^{\prime \prime}\right)$ & 2 & 5 & 3 & 1 & 4 & 12 & -17 & $\left(\mathrm{~L} .19^{\prime \prime}\right)$ & 2 & 3 & 5 & 12 & -17 & 1 & 4 \\
$\left(\mathrm{~L} .4^{\prime \prime}\right)$ & 2 & 5 & 3 & 5 & -6 & 8 & -11 & $\left(\mathrm{~L} .20^{\prime \prime}\right)$ & 2 & 3 & 5 & 8 & -11 & 5 & -6 \\
$\left(\mathrm{~L} .5^{\prime \prime}\right)$ & 2 & 5 & 3 & 8 & -15 & -1 & 4 & $\left(\mathrm{~L} .21^{\prime \prime}\right)$ & 2 & 3 & 5 & -1 & 4 & 8 & -15 \\
$\left(\mathrm{~L} .6^{\prime \prime}\right)$ & 2 & 5 & 3 & 4 & -5 & 3 & -2 & $\left(\mathrm{~L} .22^{\prime \prime}\right)$ & 2 & 3 & 5 & 3 & -2 & 4 & -5 \\
$\left(\mathrm{~L} .7^{\prime \prime}\right)$ & 2 & 5 & 3 & -1 & 10 & 6 & -7 & $\left(\mathrm{~L} .23^{\prime \prime}\right)$ & 2 & 3 & 5 & 6 & -7 & -1 & 10 \\
$\left(\mathrm{~L} .8^{\prime \prime}\right)$ & 2 & 5 & 3 & 3 & 0 & 2 & -1 & $\left(\mathrm{~L} .24^{\prime \prime}\right)$ & 2 & 3 & 5 & 2 & -1 & 3 & 0 \\
$\left(\mathrm{~L} .9^{\prime \prime}\right)$ & 2 & -1 & 1 & 0 & -1 & 1 & 0 & $\left(\mathrm{~L} .25^{\prime \prime}\right)$ & 2 & 1 & -1 & 1 & 0 & 0 & -1 \\
$\left(\mathrm{~L} .10^{\prime \prime}\right)$ & 2 & -1 & 1 & -4 & -3 & 5 & -2 & $\left(\mathrm{~L} .26^{\prime \prime}\right)$ & 2 & 1 & -1 & 5 & -2 & -4 & -3 \\
$\left(\mathrm{~L} .11^{\prime \prime}\right)$ & 2 & -1 & 1 & 2 & 1 & 7 & -2 & $\left(\mathrm{~L} .27^{\prime \prime}\right)$ & 2 & 1 & -1 & 7 & -2 & 2 & 1 \\
$\left(\mathrm{~L} .12^{\prime \prime}\right)$ & 2 & -1 & 1 & -2 & -1 & 11 & -4 & $\left(\mathrm{~L} .28^{\prime \prime}\right)$ & 2 & 1 & -1 & 11 & -4 & -2 & -1 \\
$\left(\mathrm{~L} .13^{\prime \prime}\right)$ & 2 & -1 & 1 & 3 & 2 & -8 & 3 & $\left(\mathrm{~L} .29^{\prime \prime}\right)$ & 2 & 1 & -1 & -8 & 3 & 3 & 2 \\
$\left(\mathrm{~L} .14^{\prime \prime}\right)$ & 2 & -1 & 1 & -1 & 0 & -4 & 1 & $\left(\mathrm{~L} .30^{\prime \prime}\right)$ & 2 & 1 & -1 & -4 & 1 & -1 & 0 \\
$\left(\mathrm{~L} .15^{\prime \prime}\right)$ & 2 & -1 & 1 & 5 & 4 & -2 & 1 & $\left(\mathrm{~L} .31^{\prime \prime}\right)$ & 2 & 1 & -1 & -2 & 1 & 5 & 4 \\
$\left(\mathrm{~L} .16^{\prime \prime}\right)$ & 2 & -1 & 1 & 1 & 2 & 2 & -1 & $\left(\mathrm{~L} .32^{\prime \prime}\right)$ & 2 & 1 & -1 & 2 & -1 & 1 & 2 \\
\hline
\end{tabular}

It is worth mentioning that the Toeplitz matrices constructed by conditions (L.1) - (L.16) are the transpose of the Toeplitz matrices constructed by conditions (L.17) - (L.32), respectively.

Proof of Corollary 3.7. We need only prove the necessity. Suppose that $d_{n}=$ $\operatorname{det} T_{\alpha, \beta}(n)=L_{n}$. By Theorem 3.3, it follows that $d_{0}=\gamma, d_{1}=\gamma^{2}-\alpha_{1} \beta_{1}$, and for all $n \geq 2, d_{n}=D_{1} d_{n-1}+D_{2} d_{n-2}$, where

$$
D_{1}=\gamma\left(1+A_{1} B_{1}-A_{2} B_{2}\right)-\alpha_{1} B_{1}-\beta_{1} A_{1},
$$

and

$$
D_{2}=-\left(\alpha_{1}-A_{1} \gamma-A_{2} \beta_{1}\right)\left(\beta_{1}-B_{2} \alpha_{1}-B_{1} \gamma\right)
$$

Note that, by our assumption we have $D_{1}=D_{2}=1$. Since $d_{0}=L_{0}$, we conclude that $\gamma=2$. Moreover, from $d_{1}=L_{1}$, it follows that $\gamma^{2}-\alpha_{1} \beta_{1}=1$, and since $\gamma=2$, we deduce that $\alpha_{1} \beta_{1}=3$, which implies that $\left(\alpha_{1}, \beta_{1}\right) \in\{(3,1),(1,3),(-3,-1),(-1,-3)\}$.

Suppose first that $\alpha_{1}=3$ and $\beta_{1}=1$. In this case, we observe that $\alpha_{2}=3 A_{1}+2 A_{2}$ and $\beta_{2}=B_{1}+2 B_{2}$. 
But then, the equality $3=d_{2}=\operatorname{det} T_{\alpha, \beta}(3)$ implies that $\beta_{2}=\left(7-\alpha_{2}\right) /\left(9-2 \alpha_{2}\right)$, which must be an integer. On the other hand, since $9-2 \alpha_{2}=2\left(7-\alpha_{2}\right)-5$, it follows that $9-2 \alpha_{2}$ divides 5 . So we obtain $9-2 \alpha_{2} \in\{ \pm 1, \pm 5\}$, or equivalently

$$
\alpha_{2}=3 A_{1}+2 A_{2} \in\{3 \pm 1,6 \pm 1\} \text { and } \beta_{2}=B_{1}+2 B_{2} \in\{2 \pm 1,-1 \pm 1\}
$$

Finally, if the values of $\alpha_{1}$ and $\beta_{1}$ are substituted in Eq. (3.7), then we get

$$
\left(2 A_{1}+A_{2}-3\right)\left(1-3 B_{2}-2 B_{1}\right)=1 .
$$

Solving the Eq. (3.9) in $\mathbb{Z}$, we obtain that

$$
\left(2 A_{1}+A_{2}, 3 B_{2}+2 B_{1}\right) \in\{(4,0),(2,2)\} .
$$

Thus, combining Eqs. (3.8) and (3.10), the following results are obtained:

\begin{tabular}{cccclrrrr}
\hline$\alpha_{2}$ & $\beta_{2}$ & $2 A_{1}+A_{2}$ & $2 B_{1}+3 B_{2}$ & & $A_{1}$ & $A_{2}$ & $B_{1}$ & $B_{2}$ \\
\hline 4 & 3 & 2 & 2 & $\Longrightarrow$ & 0 & 2 & -5 & 4 \\
4 & 3 & 4 & 0 & $\Longrightarrow$ & 4 & -4 & -9 & 6 \\
5 & -2 & 2 & 2 & $\Longrightarrow$ & -1 & 4 & 10 & -6 \\
5 & -2 & 4 & 0 & $\Longrightarrow$ & 3 & -2 & 6 & -4 \\
2 & 1 & 4 & 0 & $\Longrightarrow$ & 6 & -8 & -3 & 2 \\
2 & 1 & 2 & 2 & $\Longrightarrow$ & 2 & -2 & 1 & 0 \\
7 & 0 & 2 & 2 & $\Longrightarrow$ & -3 & 8 & 4 & -2 \\
7 & 0 & 4 & 0 & $\Longrightarrow$ & 1 & 2 & 0 & 0
\end{tabular}

The remaining proofs for the cases $\left(\alpha_{1}, \beta_{1}\right) \in\{(1,3),(-3,-1),(-1,-3)\}$ are exactly as in the previous case. This concludes the part $(a)$.

(b) This follows directly from part (a), Theorem 2.4, and Lemma $3.2(a)$.

(c) This follows from part (a), Lemma $3.2(a)$ and Eq. (2.3).

Corollary 3.8. Let $A_{1}, A_{2}, B_{1}, B_{2}$ and $r$ be integers with $r \geq 1$. Let $\alpha=\left(\alpha_{i}\right)_{i \geq 0}$ and $\beta=\left(\beta_{i}\right)_{i \geq 0}$ be two integer sequences satisfy $\alpha_{0}=\beta_{0}=\gamma$ and linear recurrences

$$
\alpha_{i}=A_{1} \alpha_{i-1}+A_{2} \alpha_{i-2} \quad \text { and } \quad \beta_{i}=B_{1} \beta_{i-1}+B_{2} \beta_{i-2} \quad \text { for all } i \geq 2 \text {. }
$$

Then, it holds that 
(a) For any nonnegative integer $n$, $\operatorname{det} T_{\alpha, \beta}(n)=G_{n}^{(0,1, r, 1)}$ if and only if $\gamma, \alpha_{1}$, $\beta_{1}, A_{1}, A_{2}, B_{1}$ and $B_{2}$ satisfy one of the following conditions, where $c$ is a constant.

\begin{tabular}{|l|ccccccc|}
\hline & $\gamma$ & $\alpha_{1}$ & $\beta_{1}$ & $A_{1}$ & $A_{2}$ & $B_{1}$ & $B_{2}$ \\
\hline (FP.1) & 0 & 1 & -1 & $c+r$ & 0 & $c$ & 0 \\
$(\mathrm{FP} .2)$ & 0 & 1 & -1 & $c+r$ & -2 & $c$ & -2 \\
$(\mathrm{FP} .3)$ & 0 & -1 & 1 & $c$ & 0 & $c+r$ & 0 \\
$(\mathrm{FP} .4)$ & 0 & -1 & 1 & $c$ & -2 & $c+r$ & -2 \\
\hline
\end{tabular}

(b) For any nonnegative integer $n, \operatorname{det} A_{\alpha, \beta}(n)=G_{n}^{(0,1, r, 1)}$ if and only if $\gamma, \alpha_{1}$, $\beta_{1}, A_{1}, A_{2}, B_{1}$ and $B_{2}$ satisfy one of the following conditions, where $c$ is a constant.

\begin{tabular}{|c|ccccccc|}
\hline & $\gamma$ & $\alpha_{1}$ & $\beta_{1}$ & $A_{1}$ & $A_{2}$ & $B_{1}$ & $B_{2}$ \\
\hline$\left(\mathrm{FP} .1^{\prime}\right)$ & 0 & 1 & -1 & $c+r+2$ & $-c-r-1$ & $c$ & 0 \\
$\left(\mathrm{FP} .2^{\prime}\right)$ & 0 & 1 & -1 & $c+r+2$ & $-c-r-3$ & $c$ & -2 \\
$\left(\mathrm{FP} .3^{\prime}\right)$ & 0 & -1 & 1 & $c+2$ & $-c-1$ & $c+r$ & 0 \\
$\left(\mathrm{FP} .4^{\prime}\right)$ & 0 & -1 & 1 & $c+2$ & $-c-3$ & $c+r$ & -2 \\
\hline
\end{tabular}

(c) For any nonnegative integer $n$, $\operatorname{det} P_{\alpha, \beta}(n)=G_{n}^{(0,1, r, 1)}$ if and only if $\gamma, \alpha_{1}$, $\beta_{1}, A_{1}, A_{2}, B_{1}$ and $B_{2}$ satisfy one of the following conditions, where $c$ is a constant.

\begin{tabular}{|c|ccccccc|}
\hline & $\gamma$ & $\alpha_{1}$ & $\beta_{1}$ & $A_{1}$ & $A_{2}$ & $B_{1}$ & $B_{2}$ \\
\hline$\left(\mathrm{FP} .1^{\prime \prime}\right)$ & 0 & 1 & -1 & $c+r+2$ & $-c-r-1$ & $c+2$ & $-c-1$ \\
$\left(\mathrm{FP} .2^{\prime \prime}\right)$ & 0 & 1 & -1 & $c+r+2$ & $-c-r-3$ & $c+2$ & $-c-3$ \\
$\left(\mathrm{FP} .3^{\prime \prime}\right)$ & 0 & -1 & 1 & $c+2$ & $-c-1$ & $c+r+2$ & $-c-r-1$ \\
$\left(\mathrm{FP} .4^{\prime \prime}\right)$ & 0 & -1 & 1 & $c+2$ & $-c-3$ & $c+r+2$ & $-c-r-3$ \\
\hline
\end{tabular}

Proof. (a) The sufficiency is clear. To prove the necessity, suppose that $d_{n}=$ $\operatorname{det} T_{\alpha, \beta}(n)=G_{n}^{(0,1, r, 1)}$. By Theorem 3.3, it follows that $d_{0}=\gamma, d_{1}=\gamma^{2}-\alpha_{1} \beta_{1}$, and for all $n \geq 2, d_{n}=D_{1} d_{n-1}+D_{2} d_{n-2}$, where

$$
D_{1}=\gamma\left(1+A_{1} B_{1}-A_{2} B_{2}\right)-\alpha_{1} B_{1}-\beta_{1} A_{1},
$$

and

$$
D_{2}=-\left(\alpha_{1}-A_{1} \gamma-A_{2} \beta_{1}\right)\left(\beta_{1}-B_{2} \alpha_{1}-B_{1} \gamma\right)
$$

Note that, by our assumption we must have $D_{1}=r$ and $D_{2}=1$. Since $d_{0}=$ $G_{0}^{(0,1, r, 1)}=0$, we conclude that $\gamma=0$. Moreover, from $d_{1}=G_{1}^{(0,1, r, 1)}=1$, it 
follows that $\gamma^{2}-\alpha_{1} \beta_{1}=1$, and since $\gamma=0$, we deduce that $\alpha_{1} \beta_{1}=-1$, which implies that $\left(\alpha_{1}, \beta_{1}\right) \in\{(1,-1),(-1,1)\}$. In both cases, from $D_{2}=1$, it follows that $\left(A_{2}+1\right)\left(B_{2}+1\right)=1$, and solving this equation in $\mathbb{Z}$, one gets $\left(A_{2}, B_{2}\right) \in$ $\{(0,0),(-2,-2)\}$. Moreover, from $D_{1}=r$, it follows that $A_{1}-r=B_{1}(:=c)$, if $\left(\alpha_{1}, \beta_{1}\right)=(1,-1)$ and $A_{1}+r=B_{1}(:=c)$, if $\left(\alpha_{1}, \beta_{1}\right)=(-1,1)$.

(b) This follows directly from part (a), Theorem 2.4, and Lemma $3.2(a)$.

(c) This follows from part (a), Lemma $3.2(a)$ and Eq. (2.3).

Corollary 3.9. Let $A_{1}, A_{2}, B_{1}$ and $B_{2}$ be integers and let $s$ be a prime number. Let $\alpha=\left(\alpha_{i}\right)_{i \geq 0}$ and $\beta=\left(\beta_{i}\right)_{i \geq 0}$ be two integer sequences satisfy $\alpha_{0}=\beta_{0}=\gamma$ and linear recurrences

$$
\alpha_{i}=A_{1} \alpha_{i-1}+A_{2} \alpha_{i-2} \quad \text { and } \quad \beta_{i}=B_{1} \beta_{i-1}+B_{2} \beta_{i-2} \quad \text { for all } i \geq 2 .
$$

Then it holds that

(a) For any nonnegative integer $n$, $\operatorname{det} T_{\alpha, \beta}(n)=G_{n}^{(0,1,1, s)}$ if and only if $\gamma, \alpha_{1}$, $\beta_{1}, A_{1}, A_{2}, B_{1}$ and $B_{2}$ satisfy one of the following conditions, where $c$ is a constant.

\begin{tabular}{|l|ccccccc|}
\hline & $\gamma$ & $\alpha_{1}$ & $\beta_{1}$ & $A_{1}$ & $A_{2}$ & $B_{1}$ & $B_{2}$ \\
\hline (J.1) & 0 & 1 & -1 & $c+1$ & 0 & $c$ & $s-1$ \\
(J.2) & 0 & 1 & -1 & $c+1$ & -2 & $c$ & $-s-1$ \\
(J.3) & 0 & 1 & -1 & $c+1$ & $s-1$ & $c$ & 0 \\
(J.4) & 0 & 1 & -1 & $c+1$ & $-s-1$ & $c$ & -2 \\
(J.5) & 0 & -1 & 1 & $c$ & $s-1$ & $c+1$ & 0 \\
(J.6) & 0 & -1 & 1 & $c$ & $-s-1$ & $c+1$ & -2 \\
(J.7) & 0 & -1 & 1 & $c$ & 0 & $c+1$ & $s-1$ \\
(J.8) & 0 & -1 & 1 & $c$ & -2 & $c+1$ & $-s-1$ \\
\hline
\end{tabular}

(b) For any nonnegative integer $n$, $\operatorname{det} A_{\alpha, \beta}(n)=G_{n}^{(0,1,1, s)}$ if and only if $\gamma, \alpha_{1}$, $\beta_{1}, A_{1}, A_{2}, B_{1}$ and $B_{2}$ satisfy one of the following conditions, where $c$ is a constant.

\begin{tabular}{|c|ccccccc|}
\hline & $\gamma$ & $\alpha_{1}$ & $\beta_{1}$ & $A_{1}$ & $A_{2}$ & $B_{1}$ & $B_{2}$ \\
\hline$\left(\mathrm{J} .1^{\prime}\right)$ & 0 & 1 & -1 & $c+3$ & $-c-2$ & $c$ & $s-1$ \\
$\left(\mathrm{~J} .2^{\prime}\right)$ & 0 & 1 & -1 & $c+3$ & $-c-4$ & $c$ & $-s-1$ \\
$\left(\mathrm{~J} .3^{\prime}\right)$ & 0 & 1 & -1 & $c+3$ & $s-c-3$ & $c$ & 0 \\
$\left(\mathrm{~J} .4^{\prime}\right)$ & 0 & 1 & -1 & $c+3$ & $-s-c-3$ & $c$ & -2 \\
$\left(\mathrm{~J} .5^{\prime}\right)$ & 0 & -1 & 1 & $c+2$ & $s-c-2$ & $c+1$ & 0 \\
$\left(\mathrm{~J} .6^{\prime}\right)$ & 0 & -1 & 1 & $c+2$ & $-s-c-2$ & $c+1$ & -2 \\
$\left(\mathrm{~J} .7^{\prime}\right)$ & 0 & -1 & 1 & $c+2$ & $-c-1$ & $c+1$ & $s-1$ \\
$\left(\mathrm{~J} .8^{\prime}\right)$ & 0 & -1 & 1 & $c+2$ & $-c-3$ & $c+1$ & $-s-1$ \\
\hline
\end{tabular}


(c) For any nonnegative integer $n$, $\operatorname{det} P_{\alpha, \beta}(n)=G_{n}^{(0,1,1, s)}$ if and only if $\gamma, \alpha_{1}$, $\beta_{1}, A_{1}, A_{2}, B_{1}$ and $B_{2}$ satisfy one of the following conditions, where $c$ is a constant.

\begin{tabular}{|c|ccccccc|}
\hline & $\gamma$ & $\alpha_{1}$ & $\beta_{1}$ & $A_{1}$ & $A_{2}$ & $B_{1}$ & $B_{2}$ \\
\hline$\left(\mathrm{J} .1^{\prime \prime}\right)$ & 0 & 1 & -1 & $c+3$ & $-c-2$ & $c+2$ & $s-c-2$ \\
$\left(\mathrm{~J} .2^{\prime \prime}\right)$ & 0 & 1 & -1 & $c+3$ & $-c-4$ & $c+2$ & $-s-c-2$ \\
$\left(\mathrm{~J} .3^{\prime \prime}\right)$ & 0 & 1 & -1 & $c+3$ & $s-c-3$ & $c+2$ & $-c-1$ \\
$\left(\mathrm{~J} .4^{\prime \prime}\right)$ & 0 & 1 & -1 & $c+3$ & $-s-c-3$ & $c+2$ & $-c-3$ \\
$\left(\mathrm{~J} .5^{\prime \prime}\right)$ & 0 & -1 & 1 & $c+2$ & $s-c-2$ & $c+3$ & $-c-2$ \\
$\left(\mathrm{~J} .6^{\prime \prime}\right)$ & 0 & -1 & 1 & $c+2$ & $-s-c-2$ & $c+3$ & $-c-4$ \\
$\left(\mathrm{~J} .7^{\prime \prime}\right)$ & 0 & -1 & 1 & $c+2$ & $-c-1$ & $c+3$ & $s-c-3$ \\
$\left(\mathrm{~J} .8^{\prime \prime}\right)$ & 0 & -1 & 1 & $c+2$ & $-c-3$ & $c+3$ & $-s-c-3$ \\
\hline
\end{tabular}

Proof. (a) The sufficiency is clear. To prove the necessity, suppose that $d_{n}=$ $\operatorname{det} T_{\alpha, \beta}(n)=G_{n}^{(0,1,1, s)}$. By Theorem 3.3, it follows that $d_{0}=\gamma, d_{1}=\gamma^{2}-\alpha_{1} \beta_{1}$, and for all $n \geq 2, d_{n}=D_{1} d_{n-1}+D_{2} d_{n-2}$, where

$$
D_{1}=\gamma\left(1+A_{1} B_{1}-A_{2} B_{2}\right)-\alpha_{1} B_{1}-\beta_{1} A_{1},
$$

and

$$
D_{2}=-\left(\alpha_{1}-A_{1} \gamma-A_{2} \beta_{1}\right)\left(\beta_{1}-B_{2} \alpha_{1}-B_{1} \gamma\right)
$$

Note that, by our assumption we must have $D_{1}=1$ and $D_{2}=s$. Since $d_{0}=$ $G_{0}^{(0,1,1, s)}=0$, we conclude that $\gamma=0$. Moreover, from $d_{1}=G_{1}^{(0,1,1, s)}=1$, it follows that $\gamma^{2}-\alpha_{1} \beta_{1}=1$, and since $\gamma=0$, we deduce that $\alpha_{1} \beta_{1}=-1$, which implies that $\left(\alpha_{1}, \beta_{1}\right) \in\{(1,-1),(-1,1)\}$. In both cases, we have $D_{2}=\left(A_{2}+1\right)\left(B_{2}+1\right)=s$, and since $s$ is a prime number, we obtain

$$
\left(A_{2}+1, B_{2}+1\right) \in\{(1, s),(-1,-s),(s, 1),(-s,-1)\},
$$

or equivalently

$$
\left(A_{2}, B_{2}\right) \in\{(0, s-1),(-2,-s-1),(s-1,0),(-s-1,-2)\} .
$$

Furthermore, in the case that $\left(\alpha_{1}, \beta_{1}\right)=(1,-1)$, from $D_{1}=1$ we conclude that $A_{1}-1=B_{1}(:=c)$, and if $\left(\alpha_{1}, \beta_{1}\right)=(-1,1)$, we get $A_{1}+1=B_{1}(:=c)$. The proof of part $(a)$ is now complete.

(b) This follows directly from part $(a)$, Theorem 2.4, and Lemma $3.2(a)$. 
(c) This follows from part (a), Lemma $3.2(a)$ and Eq. (2.3).

REMARK 3.10. Although in Corollaries 3.8 and 3.9, we have restricted ourselves to integer values, we should mention that the necessary part still holds for all values of $c$ (not necessarily an integer) in the above conditions. Also, note that some of the matrices constructed by the above conditions are the transpose of each other. In addition, by Corollaries 3.8 and 3.9, there exist some infinite families of integer matrices whose leading principal minors form the Fibonacci, Pell and Jacobsthal sequences (for $r=1,2$ in Theorem 3.8 and $s=2$ in Theorem 3.9, respectively).

At last, we assume that $d_{n}=\operatorname{det} P_{\alpha, \beta}(n)$, where $\alpha$ and $\beta$ are $r$-recurrent and $s$-recurrent, respectively, starting with a common first term. In [1], Bacher proved that if both $\alpha$ and $\beta$ are 2-recurrent then so $\left(d_{n}\right)_{n \geq 0}$ (see Theorem 3.1 in [1]). In the same article, he conjectured that the sequence $\left(\bar{d}_{n}\right)_{n \geq 0}$ is a $\left(\begin{array}{c}r+s-2 \\ r-1\end{array}\right)$-recurrent, in general. This conjecture was later proved by Petkovšek and Zakrajšek in [15]. Here, we provide another proof of Theorem 3.1 in [1].

THEOREM 3.11. Let $\alpha, \beta$ be 2-recurrents starting with a common first term and $d_{n}=\operatorname{det} P_{\alpha, \beta}(n)$. Then the sequence $\left(d_{n}\right)_{n \geq 0}$ is also a 2-recurrent.

Proof. From Eq. (2.1), we have $P_{\alpha, \beta}(n)=L(n) \cdot T_{\hat{\alpha}, \hat{\beta}}(n) \cdot U(n)$, which implies that $d_{n}=\operatorname{det} T_{\hat{\alpha}, \hat{\beta}}(n)$. Using Theorem 3.3 and Lemma $3.2(b)$, the assertion immediately follows.

Acknowledgment. We would like to thank the referee for the careful reading of an earlier version of this manuscript.

\section{REFERENCES}

[1] R. Bacher. Determinants of matrices related to the Pascal triangle. J. Théor. Nombres Bordeaux, 14:19-41, 2002.

[2] P.F. Byrd. Problem B-12: A Lucas determinant. Fibonacci Quart., 1(4):78, 1963.

[3] N.D. Cahill, J.R. D'Errico, D.A. Narayan, and J.Y. Narayan. Fibonacci determinants. College Math. J., 33(3):221-225, 2002.

[4] N.D. Cahill, J.R. D'Errico, and J.P. Spence. Complex factorizations of the Fibonacci and Lucas numbers. Fibonacci Quart., 41(1):13-19, 2003.

[5] N.D. Cahill and D.A. Narayan. Fibonacci and Lucas numbers as tridiagonal matrix determinants. Fibonacci Quart., 42(3):216-221, 2004.

[6] G.S. Cheon, S.G. Hwang, S.H. Rim, and S.Z. Song. Matrices determined by a linear recurrence relation among entries. Linear Algebra Appl., 373:89-99, 2003.

[7] K. Griffin, J.L. Stuart, and M.J. Tsatsomeros. Noncirculant Toeplitz matrices all of whose powers are Toeplitz. Czechoslovak Math. J., 58(4):1185-1193, 2008.

[8] C. Krattenthaler. Advanced determinant calculus. Sém. Lothar. Combin., 42: Article B42q, 1999.

[9] C. Krattenthaler. Advanced determinant calculus: a complement. Linear Algebra Appl., 411:68-166, 2005. 
[10] A.R. Moghaddamfar and S.M.H. Pooya. Generalized Pascal triangles and Toeplitz matrices. Electron. J. Linear Algebra, 18:564-588, 2009.

[11] A.R. Moghaddamfar, S.M.H. Pooya, S. Navid Salehy, and S. Nima Salehy. Fibonacci and Lucas sequences as the principal minors of some infinite matrices. J. Algebra Appl., 8(6):869-883, 2009.

[12] G. Strang. Introduction to Linear Algebra, 3rd edition. Wellesley-Cambridge Press, 2003.

[13] G. Strang and K. Borre. Linear Algebra, Geodesy, and GPS. Wellesley-Cambridge Press, 1997.

[14] M. Tan. Matrices associated to biindexed linear recurrence relations. Ars. Combin., 86:305-319, 2008.

[15] H. Zakrajšek and M. Petkovšek. Pascal-like determinants are recursive. Adv. in Appl. Math., 33(3):431-450, 2004. 